\title{
ULTRA REGULAR COVERING SPACE AND ITS AUTOMORPHISM GROUP
}

\author{
SANG-EON HAN \\ Institute of Pure and Applied Mathematics \\ Faculty of Liberal Education, Chonbuk National University, Jeonju-City Jeonbuk, 561-756, South Korea \\ e-mail: sehanejbnu.ac.kr
}

\begin{abstract}
In order to classify digital spaces in terms of digital-homotopic theoretical tools, a recent paper by Han (2006b) (see also the works of Boxer and Karaca (2008) as well as Han (2007b)) established the notion of regular covering space from the viewpoint of digital covering theory and studied an automorphism group (or Deck's discrete transformation group) of a digital covering. By using these tools, we can calculate digital fundamental groups of some digital spaces and classify digital covering spaces satisfying a radius 2 local isomorphism (Boxer and Karaca, 2008; Han, 2006b; 2008b; 2008d; 2009b). However, for a digital covering which does not satisfy a radius 2 local isomorphism, the study of a digital fundamental group of a digital space and its automorphism group remains open. In order to examine this problem, the present paper establishes the notion of an ultra regular covering space, studies its various properties and calculates an automorphism group of the ultra regular covering space. In particular, the paper develops the notion of compatible adjacency of a digital wedge. By comparing an ultra regular covering space with a regular covering space, we can propose strong merits of the former.
\end{abstract}

Keywords: digital image, digital isomorphism, (ultra) regular covering space, digital covering space, simply $k$-connected, Deck's discrete transformation group, compatible adjacency, digital wedge, automorphism group.

\section{Introduction}

Let $\mathbb{N}, \mathbb{Z}$ and $\mathbb{R}$ denote the sets of natural numbers, integers and real numbers, respectively. Let $\mathbb{Z}^{n}$ denote the set of points in the $n$-dimensional Euclidean space with integer coordinates. Useful tools from algebraic topology and geometric topology for studying digital topological properties of a (binary) digital space include a digital covering space, a (digital) $k$-fundamental group, a digital $k$-surface and so forth. These have been studied in numerous papers (Boxer, 1999; Boxer and Karaca, 2008; Han, 2005b; 2005c; 2005d; 2006a; 2006b; 2006c; 2006d; $2007 \mathrm{a} ; 2007 \mathrm{~b} ; 2008 \mathrm{a} ; 2008 \mathrm{~b}$; 2008c; 2008d; 2009a; 2009b; 2009c; 2010a; 2010b; 2010c; Malgouyres and Lenoir, 2000; Khalimsky, 1987; Rosenfeld and Klette, 2003).

Motivated by a regular covering space in algebraic topology (Spanier, 1966), its digital version was established in digital covering theory (Han, 2006b) (see also Han, 2007b), which plays an important role in classifying digital covering spaces (Boxer and Karaca, 2008; Han, 2010a). In algebraic topology, for a circle $S^{1}$ the existence problem of its regular covering space has substantially contributed to the study of a covering space in topology (Massey, 1977; Spanier, 1966). It is well known that for a covering $(\tilde{X}, p, X)$, if the classical fundamental group $\pi_{1}(X)$ is an abelian group, then the total space $\tilde{X}$ is regular (Massey, 1977; Spanier, 1966). Unlike these properties, their digital versions have some intrinsic features (Han, 2006b; 2007b; 2009a; 2009b; 2009c; 2010a; 2010b; 2010c; In-Soo Kim and Han, 2008). Boxer and Karaca (2008) as well as Han (2006b; 2007b; 2008a; 2008b; 2009b) studied an automorphism group of a radius 2 (digital) covering $(E, p, B)$. In addition, Boxer and Karaca (2008) studied a classification of digital spaces by using the conjugacy class corresponding to a digital covering. Furthermore, Han (2009c) developed the generalized universal (briefly, GU-)covering property which improves the universal $(2, k)$-covering property of Boxer (2006).

Main applications of digital covering theory include the calculations of both a digital fundamental group of a digital space and an automorphism group of a digital covering. For many digital coverings $(E, p, B)$ except that satisfying a radius 2 local isomorphism the study of both their automorphism groups and digital fundamental groups of $E$ and $B$ remains open. In order to answer this query, the paper establishes the notion of an ultra regular 
digital covering space and studies an automorphism group of a digital covering $(E, p, B)$ which does not satisfy a radius 2 local isomorphism. This can play an important role in classifying digital covering spaces.

The paper is organized as follows. Section 2 provides some basic notions. Section 3 reviews some results related to the study of a radius 2 (digital) covering and investigates some properties of a regular covering space from the viewpoint of digital covering theory. Section 4 develops the notion of compatible $k$-adjacency of a digital wedge which can be used for studying an automorphism group of an ultra regular covering space in Section 5. In addition, we discuss a limitation of the digital version of a regular covering in algebraic topology. Section 5 develops the notion of an ultra regular covering space and studies automorphism groups of several kinds of digital coverings. Section 6 compares an ultra regular covering space and a regular covering space, and refers to strong merits of the former. Finally, Section 7 concludes the paper with a summary.

\section{Preliminaries}

To study a multidimensional digital space $X \subset \mathbb{Z}^{n}$, let us now recall the $k$-adjacency relations of $\mathbb{Z}^{n}$ as well as some essential terminology such as a digital isomorphism, a digital homotopy, a strong $k$-deformation retract and so forth. Motivated by the $k$-adjacency relations of $2 D$ and $3 D$ digital spaces (Kong and Rosenfeld, 1996; Rosenfeld, $1979)$, the $k$-adjacency relations of $\mathbb{Z}^{n}$ were established (Han, 2003) (see also Han, 2005c; 2008d; 2010c). For a natural number $m$ with $1 \leq m \leq n$, two distinct points

$$
p=\left(p_{1}, p_{2}, \cdots, p_{n}\right), q=\left(q_{1}, q_{2}, \cdots, q_{n}\right) \in \mathbb{Z}^{n}
$$

are called $k(m, n)$ - (briefly, $k$-) adjacent if

- there are at most $m$ indices $i$ such that $\left|p_{i}-q_{i}\right|=1$ and

- for all other indices $i$ such that $\left|p_{i}-q_{i}\right| \neq 1, p_{i}=q_{i}$.

Concretely, we can observe that the $k(m, n)$ (or $k$ )adjacency relations of $\mathbb{Z}^{n}$ are determined according to the two numbers $m, n \in \mathbb{N}$ (Han, 2003) (also Han, 2005c; 2008d; 2010c), as follows.

Proposition 1. (Han, 2008d) By using the above operator, we can obtain the $k$-adjacency of $\mathbb{Z}^{n}$ as follows:

$$
k:=k(m, n)=\sum_{i=n-m}^{n-1} 2^{n-i} C_{i}^{n},
$$

where

$$
C_{i}^{n}=\frac{n !}{(n-i) ! i !}
$$

In general, a pair $(X, k)$ is assumed to be a (binary) digital space (or digital image) with $k$-adjacency in a quadruple $\left(\mathbb{Z}^{n}, k, \bar{k}, X\right)$, where $(k, \bar{k}) \in\{(k, 2 n),(2 n$, $\left.\left.3^{n}-1\right)\right\}$ with $k \neq \bar{k}, k$ represents an adjacency relation for $X$, and $\bar{k}$ represents an adjacency relation for $\mathbb{Z}^{n}-X$ (Kong and Rosenfeld, 1996). More precisely, owing to the digital $k$-connectivity paradox found in the work of Kong and Rosenfeld (1996), we remind $k \neq \bar{k}$ except the case $(\mathbb{Z}, 2,2, X)$. For $\{a, b\} \subset \mathbb{Z}$ with $a \leq b,[a, b]_{\mathbb{Z}}=$ $\{a \leq n \leq b \mid n \in \mathbb{Z}\}$ is considered in $\left(\mathbb{Z}, 2,2,[a, b]_{\mathbb{Z}}\right)$ (Boxer, 1999). But in this paper we are not concerned with $\bar{k}$-adjacency between two points in $\mathbb{Z}^{n}-X$.

We say that two subsets $(A, k)$ and $(B, k)$ of $(X, k)$ are $k$-adjacent to each other if $A \cap B=\emptyset$ and there are points $a \in A$ and $b \in B$ such that $a$ and $b$ are $k$-adjacent to each other (Kong and Rosenfeld, 1996). We say that a set $X \subset \mathbb{Z}^{n}$ is $k$-connected if it is not a union of two disjoint non-empty sets that are not $k$-adjacent to each other (Kong and Rosenfeld, 1996). For an adjacency relation $k$ of $\mathbb{Z}^{n}$, a simple $k$-path with $l+1$ elements in $\mathbb{Z}^{n}$ is assumed to be an injective sequence $\left(x_{i}\right)_{i \in[0, l]_{\mathbb{Z}}} \subset \mathbb{Z}^{n}$ such that $x_{i}$ and $x_{j}$ are $k$-adjacent if and only if either $j=i+1$ or $i=j+1$ (Kong and Rosenfeld, 1996). If $x_{0}=x$ and $x_{l}=y$, then we say that the length of the simple $k$-path, denoted by $l_{k}(x, y)$, is the number $l$. A simple closed $k$-curve with $l$ elements in $\mathbb{Z}^{n}$, denoted by $S C_{k}^{n, l}$ (Han, 2006b), is the simple $k$-path $\left(x_{i}\right)_{i \in[0, l-1]_{\mathbb{Z}}}$, where $x_{i}$ and $x_{j}$ are $k$-adjacent if and only if $j=i+1(\bmod l)$ or $i=j+1(\bmod l)$ (Kong and Rosenfeld, 1996).

In the study of digital continuity and various properties of a digital space (Han, 2006a; 2006d), we have often used the following digital $k$-neighborhood of a point $x \in X$ with radius $\varepsilon \in \mathbb{N}$ (Han, 2003) (see also Han, 2005c): For a digital space $(X, k)$ in $\mathbb{Z}^{n}$, the digital $k$-neighborhood of $x_{0} \in X$ with radius $\varepsilon$ is defined in $X$ to be the following subset of $X: N_{k}\left(x_{0}, \varepsilon\right)=\{x \in$ $\left.X \mid l_{k}\left(x_{0}, x\right) \leq \varepsilon\right\} \cup\left\{x_{0}\right\}$, where $l_{k}\left(x_{0}, x\right)$ is the length of a shortest simple $k$-path from $x_{0}$ to $x$ and $\varepsilon \in \mathbb{N}$.

Motivated by both the digital continuity of Rosenfeld (1979) and the $\left(k_{0}, k_{1}\right)$-continuity of Boxer (1999), we can present digital continuity which can be substantially used for studying digital spaces in $\mathbb{Z}^{n}, n \in \mathbb{N}$, as follows.

Proposition 2. (Han, 2008d) Let $\left(X, k_{0}\right)$ and $\left(Y, k_{1}\right)$ be digital spaces in $\mathbb{Z}^{n_{0}}$ and $\mathbb{Z}^{n_{1}}$, respectively. A function $f: X \rightarrow Y$ is $\left(k_{0}, k_{1}\right)$-continuous if and only if for every $x \in X, f\left(N_{k_{0}}(x, 1)\right) \subset N_{k_{1}}(f(x), 1)$.

Since a digital space $(X, k)$ can be considered to be a digital $k$-graph, we may use the term a $\left(k_{0}, k_{1}\right)$ isomorphism as in the work of Han (2005d) (see also Boxer, 2006) rather than a $\left(k_{0}, k_{1}\right)$-homeomorphism as used by Boxer (1999), as follows.

Definition 1. (Han, 2005d, see also Boxer, 2006) For two digital spaces $\left(X, k_{0}\right)$ in $\mathbb{Z}^{n_{0}}$ and $\left(Y, k_{1}\right)$ in $\mathbb{Z}^{n_{1}}$, a 
map $h: X \rightarrow Y$ is called a $\left(k_{0}, k_{1}\right)$-isomorphism if $h$ is a $\left(k_{0}, k_{1}\right)$-continuous bijection and, further, $h^{-1}$ : $Y \rightarrow X$ is $\left(k_{1}, k_{0}\right)$-continuous. Then we use the notation $X \approx_{\left(k_{0}, k_{1}\right)} Y$. If $n_{0}=n_{1}$ and $k_{0}=k_{1}$, then we speak about a $k_{0}$-isomorphism and use the notation $X \approx_{k_{0}} Y$.

For a digital space $(X, k)$ and $A \subset X,(X, A)$ is called a digital space pair with $k$-adjacency (Han, 2006a). Furthermore, if $A$ is a singleton set $\left\{x_{0}\right\}$, then $\left(X, x_{0}\right)$ is called a pointed digital space (Kong and Rosenfeld, 1996). Based on the pointed digital homotopy of Boxer (1999), the following notion of $k$-homotopy relative to a subset $A \subset X$ has been often used in studying a $k$ homotopic thinning and a strong $k$-deformation retract of a digital space $(X, k)$ in $\mathbb{Z}^{n}$ (Han, 2008d).

Definition 2. (Han, 2006a, see also Han, 2006b; 2007a) Let $\left((X, A), k_{0}\right)$ and $\left(Y, k_{1}\right)$ be a digital space pair and a digital space, respectively. Let $f, g: X \rightarrow Y$ be $\left(k_{0}, k_{1}\right)$ continuous functions. Suppose there exist $m \in \mathbb{N}$ and a function $F: X \times[0, m]_{\mathbb{Z}} \rightarrow Y$ such that

- For all $x \in X, F(x, 0)=f(x)$ and $F(x, m)=g(x)$.

- For all $x \in X$, the induced function $F_{x}:[0, m]_{\mathbb{Z}} \rightarrow$ $Y$ given by $F_{x}(t)=F(x, t)$ for all $t \in[0, m]_{\mathbb{Z}}$ is $\left(2, k_{1}\right)$-continuous.

- For all $t \in[0, m]_{\mathbb{Z}}$, the induced function $F_{t}$ : $X \rightarrow Y$ given by $F_{t}(x)=F(x, t)$ for all $x \in X$ is $\left(k_{0}, k_{1}\right)$-continuous. Then we say that $F$ is a $\left(k_{0}, k_{1}\right)$-homotopy between $f$ and $g$ (Boxer, 1999).

- Furthermore, for all $t \in[0, m]_{\mathbb{Z}}$ then suppose the induced map $F_{t}$ on $A$ is a constant which is a prescribed function from $A$ to $Y$. In other words, $F_{t}(x)=f(x)=g(x)$ for all $x \in A$ and for all $t \in[0, m]_{\mathbb{Z}}$. Then we call $F$ a $\left(k_{0}, k_{1}\right)$-homotopy relative to $A$ between $f$ and $g$, and we say $f$ and $g$ are $\left(k_{0}, k_{1}\right)$-homotopic relative to $A$ in $Y, f \simeq_{\left(k_{0}, k_{1}\right) \mathrm{rel} A}$ $g$ in symbols.

In Definition 2, if $A=\left\{x_{0}\right\} \subset X$, then we say that $F$ is a pointed $\left(k_{0}, k_{1}\right)$-homotopy at $\left\{x_{0}\right\}$ (Boxer, 1999). When $f$ and $g$ are pointed $\left(k_{0}, k_{1}\right)$-homotopic in $Y$, we denote by $f \simeq_{\left(k_{0}, k_{1}\right)} g$. In addition, if $k_{0}=k_{1}$ and $n_{0}=n_{1}$, then we say that $f$ and $g$ are pointed $k_{0^{-}}$ homotopic in $Y$ and use the notation $f \simeq_{k_{0}} g$ and $f \in[g]$ which means the $k_{0}$-homotopy class of $g$. If, for some $x_{0} \in X, 1_{X}$ is $k$-homotopic to the constant map with the space $x_{0}$ relative to $\left\{x_{0}\right\}$, then we say that $\left(X, x_{0}\right)$ is pointed $k$-contractible (Boxer, 1999). Indeed, the notion of $k$-contractibility is slightly different from the contractibility in Euclidean topology (Boxer, 1999) (see also Han, 2005c).

Definition 3. (Han, 2006b, see also Han, 2007a) For a digital space pair $((X, A), k)$, we say that $A$ is a strong $k$ deformation retract of $X$ if there is a digital $k$-continuous map $r$ from $X$ onto $A$ such that $F: i \circ r \simeq_{k \cdot r e l . A} 1_{X}$ and $r \circ i=1_{A}$. Then a point $x \in X-A$ is called strong $k$-deformation retractable.

By using the trivial extension presented by Boxer (1999) and the Khalimsky operation presented by Khalimsky (1987), Boxer (1999) establishes the $k$-fundamental group: For a digital space $(X, k)$, consider a $k$-loop $f$ with a base point $x_{0}$; we denote by $[f]_{X}$ (briefly, $[f]$ ) the $k$-homotopy class of $f$ in $X$. Then for a $k$-loop $f_{1}$ with the same base point $x_{0} \in X, f_{0} \in[f]$ means that the two $k$-loops $f$ and $f_{0}$ have trivial extensions that can be joined by a $k$-homotopy keeping the end point fixed (Han, 2005c) (see also Boxer, 2006). Furthermore, if $f_{1}, f_{2}, g_{1}, g_{2} \in F^{k}\left(X, x_{0}\right), f_{1} \in\left[f_{2}\right]$, and $g_{1} \in\left[g_{2}\right]$, then $f_{1} * g_{1} \in\left[f_{2} * g_{2}\right]$, i.e., $\left[f_{1} * g_{1}\right]=\left[f_{2} * g_{2}\right]$ (Boxer, 1999; Khalimsky, 1987). Then we use the notation $\pi^{k}\left(X, x_{0}\right)=\left\{[f] \mid f \in F^{k}\left(X, x_{0}\right)\right\}$ which is a group (Boxer, 1999) with the operation $[f] \cdot[g]=[f * g]$ called the (digital) $k$-fundamental group of $\left(X, x_{0}\right)$ (Boxer, 1999), where the base point is assumed to be a point which cannot be deleted by a strong $k$-deformation retract (Han, 2008a). If $X$ is pointed $k$-contractible, then $\pi^{k}\left(X, x_{0}\right)$ is trivial (Boxer, 1999).

Let $((X, A), k)$ be a digital space pair with $k$ adjacency. A map $f:\left((X, A), k_{0}\right) \rightarrow\left((Y, B), k_{1}\right)$ is called $\left(k_{0}, k_{1}\right)$-continuous if $f$ is $\left(k_{0}, k_{1}\right)$-continuous and $f(A) \subset B$ (Han, 2006a). If $A=\{a\}, B=$ $\{b\}$, we write $(X, A)=(X, a),(Y, B)=(Y, b)$, and we say that $f$ is a pointed $\left(k_{0}, k_{1}\right)$-continuous map (Kong and Rosenfeld, 1996). A $\left(k_{0}, k_{1}\right)$-continuous map $f:\left(\left(X, x_{0}\right), k_{0}\right) \rightarrow\left(\left(Y, y_{0}\right), k_{1}\right)$ induces a group homomorphism $f_{*}: \pi^{k_{0}}\left(X, x_{0}\right) \rightarrow \pi^{k_{1}}\left(Y, y_{0}\right)$ given by $f_{*}([\alpha])=[f \circ \alpha]$, where $[\alpha] \in \pi^{k_{0}}\left(X, x_{0}\right)$ (Boxer, 1999). In addition, a $\left(k_{0}, k_{1}\right)$-isomorphism $\phi$ : $\left(\left(X, x_{0}\right), k_{0}\right) \rightarrow\left(\left(Y, y_{0}\right), k_{1}\right)$ induces a group isomorphism $\phi_{*}: \pi^{k_{0}}\left(X, x_{0}\right) \rightarrow \pi^{k_{1}}\left(Y, y_{0}\right)$ (Boxer, 1999).

The following notion of "simply $k$-connected" found in the work of Han (2005c) has been often used in digital $k$-homotopy theory and digital covering theory: A pointed $k$-connected digital space $\left(X, x_{0}\right)$ is called simply $k$-connected if $\pi^{k}\left(X, x_{0}\right)$ is a trivial group.

Han (2006b) (see also Han, 2007a) proved that if $\left(A, x_{0}\right)$ is a strong $k$-deformation retract of $\left(X, x_{0}\right)$, then $\pi^{k}\left(X, x_{0}\right)$ is isomorphic to $\pi^{k}\left(A, x_{0}\right)$. Since $k$ contractibility requires a digital space $(X, k)$ to shrink $(k, k)$-continuously to a point over a finite time interval, we cannot say that $\mathbb{Z}^{n}$ is $2 n$-contractible, $n \in$ $\mathbb{N}$. However, motivated by simple 2 -connectedness of $\mathbb{Z}$ (Han, 2005c), we can obtain that $\left(\mathbb{Z}^{n}, 0_{n}\right)$ is simply $k$ connected, where $k$-adjacency is assumed to be anyone of $k$-adjacency relations of $\mathbb{Z}^{n}$.

Motivated by both 8-contractibility of $S_{8}^{2,4}$ (Boxer, 1999) and non-8-contractibility of $S C_{8}^{2,6}$ (Han, 2005c), the paper by Han (2005c) (see also Han, 2006b; 2007a) 
proved that $\pi^{k}\left(S C_{k}^{n, l}\right)$ is an infinite cyclic group, where $S C_{k}^{n, l}$ is not $k$-contractible. Precisely, $\pi^{k}\left(S C_{k}^{n, l}\right) \simeq$ $(l \mathbb{Z},+)$, where $S C_{k}^{n, l}$ is not $k$-contractible and " $\simeq$ " means a group isomorphism. In addition, $\pi^{k}\left(S C_{k}^{n, 4}\right)$ is trivial if $k=3^{n}-1, n \in \mathbb{N}-\{1\}$.

\section{Some properties of a regular covering space}

Let $(X, k)$ be a digital space in $\mathbb{Z}^{n}$. In relation to the calculation of $\pi^{k}\left(X, x_{0}\right)$ and the classification of digital spaces in terms of a digital $k$-homotopy, we have often used some properties of a digital covering (Boxer, 2006; Boxer and Karaca, 2008; Han, 2005b; 2005c; 2008d; 2009a). In digital covering theory, since each digital space $(X, k)$ is assumed to be $k$-connected, hereafter, every $(X, k)$ is considered to be $k$-connected unless stated otherwise. In this section we study some properties of a regular covering space. Let us now recall the typical axiom of a digital covering space, as follows.

Definition 4. (Han, 2005c, see also Han, 2008b) Let $\left(E, k_{0}\right)$ and $\left(B, k_{1}\right)$ be digital spaces in $\mathbb{Z}^{n_{0}}$ and $\mathbb{Z}^{n_{1}}$, respectively. Let $p: E \rightarrow B$ be a $\left(k_{0}, k_{1}\right)$-continuous surjection. Suppose that for any $b \in B$ there exists $\varepsilon \in \mathbb{N}$ such that

(1) for some index set $M, p^{-1}\left(N_{k_{1}}(b, \varepsilon)\right)=$ $\cup_{i \in M} N_{k_{0}}\left(e_{i}, \varepsilon\right)$ with $e_{i} \in p^{-1}(b)$;

(2) if $i, j \in M$ and $i \neq j$, then $N_{k_{0}}\left(e_{i}, \varepsilon\right) \cap N_{k_{0}}\left(e_{j}, \varepsilon\right)$ is an empty set; and

(3) the restriction map $p$ on $N_{k_{0}}\left(e_{i}, \varepsilon\right)$ is a $\left(k_{0}, k_{1}\right)$ isomorphism for all $i \in M$.

Then the map $p$ is called a $\left(k_{0}, k_{1}\right)$-covering map and $(E, p, B)$ is said to be a $\left(k_{0}, k_{1}\right)$-covering.

The $k_{1}$-neighborhood $N_{k_{1}}(b, \varepsilon)$ in Definition 4 is called an elementary $k_{1}$-neighborhood of $b$ with some radius $\varepsilon$ and $E$ is called a $\left(k_{0}, k_{1}\right)$-covering space of $p$.

Definition 5. (Han, 2006b, see also Han, 2008b) We say that a $\left(k_{0}, k_{1}\right)$-covering map $p:\left(E, e_{0}\right) \rightarrow\left(B, b_{0}\right)$ is an $m$-fold $\left(k_{0}, k_{1}\right)$-covering map if the cardinality of the index set $M$ is $m$.

Definition 5 can be restated as follows: For a $\left(k_{0}, k_{1}\right)$-covering map $p:\left(E, e_{0}\right) \rightarrow\left(B, b_{0}\right)$, if the set $p^{-1}\left(b_{0}\right)$ has $n$ elements (or the number $n$ can also be called the sheets of the digital covering (Massey, 1977), then the map $p$ is called an $m$-fold $\left(k_{0}, k_{1}\right)$-covering map because any points $b_{1}, b_{2} \in B$ satisfy the following identity in terms of the digital version of the corresponding properties of a covering found in the work of Massey (1977): $\sharp\left\{p^{-1}\left(b_{1}\right)\right\}=\sharp\left\{p^{-1}\left(b_{2}\right)\right\}=m$, where "\#" means the cardinality of the given set. For instance, for any $S C_{k}^{n, l}:=\left(c_{t}\right)_{t \in[0, l-1]_{\mathrm{z}}}$ we observe that $\left(S C_{k}^{n, m l}:=\left(a_{t}\right)_{t \in[0, m l-1]_{\mathbb{Z}}}, p, S C_{k}^{n, l}\right)$, given by $p\left(a_{i}\right)=$ $c_{i(\bmod l)}, m \in \mathbb{N}$, is an $m$-fold $(k, k)$-covering.
For pointed digital spaces $\left(\left(E, e_{0}\right), k_{0}\right)$ and $\left(\left(B, b_{0}\right), k_{1}\right)$, if $p:\left(E, e_{0}\right) \rightarrow\left(B, b_{0}\right)$ is a $\left(k_{0}, k_{1}\right)$ covering map such that $p\left(e_{0}\right)=b_{0}$, then the map $p$ is a pointed $\left(k_{0}, k_{1}\right)$-covering map (Han, 2005c). Hereafter, we assume that each digital covering map is a pointed one unless stated otherwise.

Definition 6. (Han, 2005a, see also Han, 2005b; 2008c) For two digital spaces $\left(X, k_{0}\right)$ in $\mathbb{Z}^{n_{0}}$ and $\left(Y, k_{1}\right)$ in $\mathbb{Z}^{n_{1}}$, a $\left(k_{0}, k_{1}\right)$-continuous map $h: X \rightarrow Y$ is called a local $\left(k_{0}, k_{1}\right)$-isomorphism if for any $x \in X, h$ maps $N_{k_{0}}(x, 1)$ $\left(k_{0}, k_{1}\right)$-isomorphically onto $N_{k_{1}}(h(x), 1) \subset Y$. If $n_{0}=$ $n_{1}$ and $k_{0}=k_{1}$, then the map $h$ is called a local $k_{0^{-}}$ isomorphism.

This local $\left(k_{0}, k_{1}\right)$-isomorphism has often been used in studying the preservation of local $k_{0}$-properties of a digital space $\left(X, k_{0}\right)$ into its corresponding $k_{1}$-ones in digital geometry (Han, 2008c).

Since a $\left(k_{0}, k_{1}\right)$-isomorphism is equivalent to a locally $\left(k_{0}, k_{1}\right)$-isomorphic bijection (Han, 2005a) (see also Han, 2006b) and a restriction map of a $\left(k_{0}, k_{1}\right)$ isomorphism is also a $\left(k_{0}, k_{1}\right)$-isomorphism (Han, 2006d), we obtain the following property: If $h$ : $\left(X, k_{0}\right) \rightarrow\left(Y, k_{1}\right)$ is a $\left(k_{0}, k_{1}\right)$-isomorphism, then the restriction map on $N_{k_{0}}(x, 1),\left.h\right|_{N_{k_{0}}(x, 1)}: N_{k_{0}}(x, 1) \rightarrow$ $N_{k_{1}}(h(x), 1)$ is a $\left(k_{0}, k_{1}\right)$-isomorphism (Han, 2005a) (see also Han, 2006d). Thus we obtain the following.

\section{Remark 1.}

(1) As discussed by Han (2006b), we may take $\varepsilon=1$ for the $\left(k_{0}, k_{1}\right)$-covering of Definition 4.

(2) As discussed by Han (2009c) (for more details, see another work of Han (2010d)), for the $\left(k_{0}, k_{1}\right)$-covering of Definition 4 we can replace " $\left(k_{0}, k_{1}\right)$-continuous surjection" with "surjection".

Definition 7. (Han, 2005b) For $n \in \mathbb{N}$, a $\left(k_{0}, k_{1}\right)$ covering $(E, p, B)$ is a radius $n$ local isomorphism if the restriction map $\left.p\right|_{N_{k_{0}}\left(e_{i}, n\right)}: N_{k_{0}}\left(e_{i}, n\right) \rightarrow N_{k_{1}}(b, n)$ is a $\left(k_{0}, k_{1}\right)$-isomorphism for all $i \in M$, where $e_{i} \in p^{-1}(b)$.

By Definition 7 , we can say that a $\left(k_{0}, k_{1}\right)$-covering $(E, p, B)$ is a radius $n$ - $\left(k_{0}, k_{1}\right)$-covering if $\varepsilon \geq n$, where the number $\varepsilon$ is the same as $\varepsilon$ in Definition 4 (Han, 2005b) (see also Han, 2008b).

In view of Definitions 4 and 7, we observe that a $\left(k_{0}, k_{1}\right)$-covering satisfying a radius $n$ local isomorphism is equivalent to a radius $n$ - $\left(k_{0}, k_{1}\right)$-covering (Han, 2006b).

Since both the unique digital lifting theorem and the digital homotopy lifting theorem will often be used for studying a digital covering space, let us now review them along with related results, as follows. For three digital spaces $\left(E, k_{0}\right)$ in $\mathbb{Z}^{n_{0}},\left(B, k_{1}\right)$ in $\mathbb{Z}^{n_{1}}$, and $\left(X, k_{2}\right)$ in $\mathbb{Z}^{n_{2}}$, let $p: E \rightarrow B$ be a $\left(k_{0}, k_{1}\right)$-continuous map. For a $\left(k_{2}, k_{1}\right)$-continuous map $f:\left(X, k_{2}\right) \rightarrow\left(B, k_{1}\right)$, as the digital analogue of the lifting found in the work 
of Massey (1977), we say that a digital lifting of $f$ is a $\left(k_{2}, k_{0}\right)$-continuous map $\tilde{f}: X \rightarrow E$ such that $p \circ \tilde{f}=f$ (Han, 2005c). We now recall the unique digital lifting theorem of Han (2005c), as follows.

Lemma 1. (Han, 2005c) For pointed digital spaces $\left(\left(E, e_{0}\right), k_{0}\right)$ in $\mathbb{Z}^{n_{0}}$ and $\left(\left(B, b_{0}\right), k_{1}\right)$ in $\mathbb{Z}^{n_{1}}$, let $p$ : $\left(E, e_{0}\right) \rightarrow\left(B, b_{0}\right)$ be a pointed $\left(k_{0}, k_{1}\right)$-covering map. Any $k_{1}$-path $f:[0, m]_{\mathbb{Z}} \rightarrow B$ beginning at $b_{0}$ has a unique digital lifting to a $k_{0}$-path $\tilde{f}$ in $E$ beginning at $e_{0}$.

Moreover, the following digital homotopy lifting theorem was introduced by Han (2005b), which plays an important role in studying digital covering theory.

Lemma 2. (Han, 2006b, see also Han, 2007b) Let $\left(\left(E, e_{0}\right), k_{0}\right)$ and $\left(\left(B, b_{0}\right), k_{1}\right)$ be pointed digital spaces. Let $p:\left(E, e_{0}\right) \rightarrow\left(B, b_{0}\right)$ be a radius $2-\left(k_{0}, k_{1}\right)$-covering map. For $k_{0}$-paths $g_{0}, g_{1}$ in $\left(E, e_{0}\right)$ that start at $e_{0}$, if there is a $k_{1}$-homotopy in $B$ from $p \circ g_{0}$ to $p \circ g_{1}$ that holds the endpoints fixed, then $g_{0}$ and $g_{1}$ have the same terminal point, and there is a $k_{0}$-homotopy in $E$ from $g_{0}$ to $g_{1}$ that holds the endpoints fixed.

The following notion has often been used for calculating the $k$-fundamental group of a digital space $(X, k)$ and classifying digital spaces (Han, 2007a; 2007b; 2008d).

Definition 8. A $\left(k_{0}, k_{1}\right)$-covering $\left(\left(E, e_{0}\right), p,\left(B, b_{0}\right)\right)$ is called regular if $p_{*} \pi^{k_{0}}\left(E, e_{0}\right)$ is a normal subgroup of $\pi^{k_{1}}\left(B, b_{0}\right)$.

As discussed by Han (2007b) (see also Han, 2008a), by using Massey's program (Massey, 1977), we obtain the following: Let $\left(\left(E, e_{0}\right), p,\left(B, b_{0}\right)\right)$ be a radius $2-\left(k_{0}, k_{1}\right)$ covering such that $E$ is $k_{0}$-connected. For any $\tilde{e} \in$ $p^{-1}\left(b_{0}\right)$ and any $\alpha \in \pi^{k_{1}}\left(B, b_{0}\right)$, defining $\tilde{e} \cdot \alpha \in p^{-1}\left(b_{0}\right)$, we observe that the set $p^{-1}\left(b_{0}\right)$ is a homogeneous right $\pi^{k_{1}}\left(B, b_{0}\right)$-space (Han, 2007b) (see also Han, 2009b) because the group $\pi^{k_{1}}\left(B, b_{0}\right)$ operates transitively on the set $p^{-1}\left(b_{0}\right)$ (Han, 2007b) (see also Han, 2008a; 2010c). Precisely, consider $e \in p^{-1}\left(b_{0}\right)$ and $\alpha \in \pi^{k_{1}}\left(B, b_{0}\right)$. Take a $k_{1}$-path $f:\left[0, m_{f}\right]_{\mathbb{Z}} \rightarrow\left(B, b_{0}\right)$ such that $[f]=\alpha$, $f(0)=b_{0}=f\left(m_{f}\right)$ Then, by Lemma 2 , the map $f$ is well-defined. By Lemma 1 , there is the unique $k_{0}$-path $\tilde{f}:\left[0, m_{f}\right]_{\mathbb{Z}} \rightarrow\left(E, e_{0}\right)$ such that $p_{*}([\tilde{f}])=[f]$ and $\tilde{f}(0)=e$ with the following.

Define

$$
p^{-1}\left(b_{0}\right) \times \pi^{k_{1}}\left(B, b_{0}\right) \rightarrow p^{-1}\left(b_{0}\right)
$$

by

$$
(e, \alpha) \rightarrow e \cdot \alpha=\tilde{f}\left(m_{f}\right),
$$

and by Lemma 2 this action is well defined because this process does not depend on the choice of the map $f$, where $[f]=\alpha$. Then for any $e \in p^{-1}\left(b_{0}\right)$, we clearly observe the following (Han, 2007b):

$$
(e, 1)=e \quad \text { and } \quad((e, \alpha), \beta)=(e, \alpha \cdot \beta),
$$

where $\alpha, \beta \in \pi^{k_{1}}\left(B, b_{0}\right)$ and 1 is the identity element. This implies $p^{-1}\left(b_{0}\right)$ admits $\pi^{k_{1}}\left(B, b_{0}\right)$ as a group of operator (or permutation).

For a $\left(k_{0}, k_{1}\right)$-covering map $p:\left(\left(E, e_{0}\right), k_{0}\right) \rightarrow$ $\left(\left(B, b_{0}\right), k_{1}\right)$ in order to study a relation between $p^{-1}\left(b_{0}\right)$ and a coset $\pi^{k_{1}}\left(B, b_{0}\right) / p_{*} \pi^{k_{0}}\left(E, e_{0}\right)$, we often use the following property.

Lemma 3. (Massey, 1977) Let $E$ be a set and $G$ a group. If $E \times G \rightarrow E$ is a transitive action, then $E$ is isomorphic to the factor group $G / G_{x_{0}}$, where $G_{x_{0}}:=\{g \in$ $\left.G \mid g\left(x_{0}\right)=x_{0}\right\}$ called the isotropy subgroup of $G$.

By using Massey's program (Massey, 1977), and Lemmas 1 and 2, we obtain the following.

Theorem 1. (Han, 2009b) Let $\left(\left(E, e_{0}\right), p,\left(B, b_{0}\right)\right)$ be a pointed radius $2-\left(k_{0}, k_{1}\right)$-covering and $\left(E, k_{0}\right) k_{0}$ connected. Then we obtain that

(1) $\pi^{k_{1}}\left(B, b_{0}\right)$ operates transitively on $p^{-1}\left(b_{0}\right)$ on the right (Han, 2007b).

(2) The right $\pi^{k_{1}}\left(B, b_{0}\right)$-space $p^{-1}\left(b_{0}\right)$ is bijective to $\pi^{k_{1}}\left(B, b_{0}\right) / p_{*} \pi^{k_{0}}\left(E, e_{0}\right)$.

Remark 2. (Han, 2009b) Let us recall that, by Theorem $5(2)$, the group $p^{-1}\left(b_{0}\right)$ found in the works of Han $(2007 b ; 2008 a ; 2008 d)$, related to the assertion of Theorem 1(2), is clearly isomorphic to the factor group $\pi^{k_{1}}\left(B, b_{0}\right) / p_{*} \pi^{k_{0}}\left(E, e_{0}\right)$ as a right $\pi^{k_{1}}\left(B, b_{0}\right)$-space, and the papers by Han $(2007 \mathrm{~b} ; 2008 \mathrm{a} ; 2008 \mathrm{~d})$ tell more about the case when $p^{-1}\left(b_{0}\right)$ has the group structure $\left(p^{-1}\left(b_{0}\right),+\right)$ derived from the given digital coverings such as $\left(\mathbb{Z}, p, S C_{k}^{n, l}\right)$ and $\left(\mathbb{Z} \times \mathbb{Z}, p_{1} \times p_{2}, S C_{k_{1}}^{n_{1}, l_{1}} \times\right.$ $\left.S C_{k_{2}}^{n_{2}, l_{2}}\right)$, where $\left(S C_{k_{1}}^{n_{1}, l_{1}} \times S C_{k_{2}}^{n_{2}, l_{2}}, k\right)$ has the $L_{S}$-or $L_{C}$-property found in the research by Han (2009a; 2010b; 2006b; 2007a; 2008d). Of course, by Theorem 1, in this case we clearly observe that the given digital covering map $p$ should be regular so that $\pi^{k_{1}}\left(B, b_{0}\right) / p_{*} \pi^{k_{0}}\left(E, e_{0}\right)$ is clearly isomorphic to $N\left(p_{*} \pi^{k_{0}}\left(E, e_{0}\right)\right) / p_{*} \pi^{k_{0}}\left(E, e_{0}\right)$.

In algebraic topology, both Deck's transformation group of a covering map and a universal covering space have strongly contributed to the study of the classification of topological spaces. Motivated by the covering homomorphism of Spanier (1966), the notion of $\left(k_{1}, k_{2}\right)$ homomorphism from a $\left(k_{1}, k\right)$-covering $\left(E_{1}, p_{1}, B\right)$ into a $\left(k_{2}, k\right)$-covering $\left(E_{2}, p_{2}, B\right)$ was introduced by Han (2007a) (see also Boxer and Karaca, 2008) and has contributed to the establishment of an automorphism group of a digital covering map in the work of Han (2008b) as well as In-Soo Kim and Han (2008), which is so different from that of a covering space in algebraic topology.

An automorphism group of a digital $\left(k_{0}, k_{1}\right)$ covering has also substantial advantages which make it convenient and efficient for calculating the digital fundamental groups of a digital spaces and classifying digital 
spaces (Han, 2006b; 2008a; 2008b, 2010b). For three digital spaces $(B, k),\left(E_{1}, k_{1}\right)$ and $\left(E_{2}, k_{2}\right)$, let $\left(E_{1}, p_{1}, B\right)$ and $\left(E_{2}, p_{2}, B\right)$ be $\left(k_{1}, k\right)$ - and $\left(k_{2}, k\right)$-coverings, respectively. Then we say that a $\left(k_{1}, k_{2}\right)$-continuous map $\phi$ : $E_{1} \rightarrow E_{2}$ such that $p_{2} \circ \phi=p_{1}$ is a $\left(k_{1}, k_{2}\right)$-covering homomorphism from $\left(E_{1}, p_{1}, B\right)$ into $\left(E_{2}, p_{2}, B\right)$ (Han, 2007a) (see also Boxer and Karaca, 2008). As a special case of this $\left(k_{1}, k_{2}\right)$-covering homomorphism, we obtain the digital version of Deck's transformation group of a covering map in algebraic topology (Spanier, 1966). Furthermore, by using the generalized universal covering property of Han (2009c), we can classify digital covering spaces.

Definition 9. (Han, 2008b, see also Boxer and Karaca, 2008; Kim and Han, 2008) Consider a $\left(k_{0}, k_{1}\right)$-covering map $p:\left(\left(E, e_{0}\right) \rightarrow\left(\left(B, b_{0}\right)\right.\right.$. A self $k_{0}$-isomorphism of the $\left(k_{0}, k_{1}\right)$-covering map $p$, denoted by $h:\left(E, k_{0}\right) \rightarrow$ $\left(E, k_{0}\right)$, is called a $k_{0}$-covering transformation or an automorphism of a digital covering map $p$ if $p=p \circ h$, where $\circ$ means the composition. The set of the automorphisms of a digital covering map with composition operation is obviously a group which is denoted by $\operatorname{Aut}(E \mid B)$ (or $A u t(E, p, B)$ ).

In the study of an automorphism group of a digital $\left(k_{0}, k_{1}\right)$-covering, by using (1) and (2), motivated by various properties of covering space found in the work of Massey (1977), we can obtain the following.

Theorem 2. (Han, 2009b)

(1) Let $\left(\left(E, e_{0}\right), p,\left(B, b_{0}\right)\right)$ be a pointed radius 2$\left(k_{0}, k_{1}\right)$-covering and $\left(E, k_{0}\right) k_{0}$-connected. Then Aut $(E \mid B)$ is isomorphic to Aut $\left(p^{-1}\left(b_{0}\right)\right)$ induced from the map $\phi \in \operatorname{Aut}(E \mid B)$, where Aut $\left(p^{-1}\left(b_{0}\right)\right)$ is considered as a right $\pi^{k_{1}}\left(B, b_{0}\right)$-space.

(2) Let $\left(\left(E, e_{0}\right), p,\left(B, b_{0}\right)\right)$ be a pointed radius 2$\left(k_{0}, k_{1}\right)$-covering and $\left(E, k_{0}\right) k_{0}$-connected. Then we obtain $\operatorname{Aut}(E \mid B) \simeq N\left(p_{*} \pi^{k_{0}}\left(E, e_{0}\right)\right) / p_{*} \pi^{k_{0}}\left(E, e_{0}\right)$, where $N\left(p_{*} \pi^{k_{0}}\left(E, e_{0}\right)\right)$ is the normalizer of $p_{*} \pi^{k_{0}}\left(E, e_{0}\right)$ in $\pi^{k_{1}}\left(B, b_{0}\right)$.

(3) Let $\left(\left(E, e_{0}\right), p,\left(B, b_{0}\right)\right)$ be a pointed radius 2$\left(k_{0}, k_{1}\right)$-covering which is regular and $\left(E, k_{0}\right) k_{0}$ connected. Then we obtain

$$
\begin{aligned}
\operatorname{Aut}(E \mid B) & \simeq \operatorname{Aut}\left(p^{-1}\left(b_{0}\right)\right) \\
& \simeq \pi^{k_{1}}\left(B, b_{0}\right) / p_{*} \pi^{k_{0}}\left(E, e_{0}\right) .
\end{aligned}
$$

(4) Let $\left(\left(E, e_{0}\right), p,\left(B, b_{0}\right)\right)$ be a pointed radius 2$\left(k_{0}, k_{1}\right)$-covering and $\left(E, k_{0}\right) k_{0}$-connected. Then we obtain the following: $\left(\left(E, e_{0}\right), p,\left(B, b_{0}\right)\right)$ is regular if and only if $p_{*} \pi^{k_{0}}\left(E, e_{0}\right)=p_{*} \pi^{k_{0}}\left(E, e_{1}\right)$, where $p\left(e_{0}\right)=$ $p\left(e_{1}\right)=b_{0}$.

By using Theorem 2, we can study automorphism groups of many digital coverings including a $\left(k_{0}, k_{1}\right)$ covering which does not satisfy a radius 2 local isomorphism in Section 5.

\section{Compatible adjacency of a digital wedge}

Since a digital wedge can play an important role in studying an automorphism group of a digital covering, let us now recall a digital wedge discussed by Han (2005c) (see also Boxer, 2006; Han, 2009c). For digital spaces $\left(X_{i}, k_{i}\right)$ in $\mathbb{Z}^{n_{i}}, i \in\{0,1\}$, the notion of digital wedge of $\left(X_{i}, k_{i}\right)$ was introduced by Han $(2005 \mathrm{c})$. In relation to the study of automorphism groups of both an ultra regular and a regular covering space in Sections 5 and 6, motivated by the former version of Han (2009c), we need to develop a notion of compatible $k$-adjacency of a digital wedge as follows.

Definition 10. For pointed digital spaces $\left(\left(X, x_{0}\right), k_{0}\right)$ in $\mathbb{Z}^{n_{0}}$ and $\left(\left(Y, y_{0}\right), k_{1}\right)$ in $\mathbb{Z}^{n_{1}}$, the wedge of $\left(X, k_{0}\right)$ and $\left(Y, k_{1}\right)$, written $\left(X \vee Y,\left(x_{0}, y_{0}\right)\right)$, is the digital space in $\mathbb{Z}^{n}$,

$$
\left\{(x, y) \in X \times Y \mid x=x_{0} \text { or } y=y_{0}\right\},
$$

with compatible $k(m, n)$ (or $k$ )-adjacency relative to both $\left(X, k_{0}\right)$ and $\left(Y, k_{1}\right)$, and the only one point $\left(x_{0}, y_{0}\right)$ in common with the following property:

(W1) The $k(m, n)$ (or $k$ )-adjacency is determined by the numbers $m$ and $n$ with $n=\max \left\{n_{0}, n_{1}\right\}, m=$ $\max \left\{m_{0}, m_{1}\right\}$ satisfying $(W 1-1)$ below, where the numbers $m_{i}$ are taken from the $k_{i}$ (or $\left.k\left(m_{i}, n_{i}\right)\right)$-adjacency relations of the given digital spaces $\left(\left(X, x_{0}\right), k_{0}\right)$ and $\left(\left(Y, y_{0}\right), k_{1}\right), i \in\{0,1\}$.

(W 1-1) In view of (3), induced from the projection maps, we can consider the natural projection maps,

$$
W_{X}:\left(X \vee Y,\left(x_{0}, y_{0}\right)\right) \rightarrow\left(X, x_{0}\right)
$$

and

$$
W_{Y}:\left(X \vee Y,\left(x_{0}, y_{0}\right)\right) \rightarrow\left(Y, y_{0}\right)
$$

In relation to the establishment of a compatible $k$ adjacency of the digital wedge $\left(X \vee Y,\left(x_{0}, y_{0}\right)\right)$, the restriction maps of $W_{X}$ and $W_{Y}$ on $\left(X \times\left\{y_{0}\right\},\left(x_{0}, y_{0}\right)\right) \subset$ $\left(X \vee Y,\left(x_{0}, y_{0}\right)\right)$ and $\left(\left\{x_{0}\right\} \times Y,\left(x_{0}, y_{0}\right)\right) \subset(X \vee$ $\left.Y,\left(x_{0}, y_{0}\right)\right)$ satisfy the following properties, respectively,

(1) $\left.W_{X}\right|_{X \times\left\{y_{0}\right\}}:\left(X \times\left\{y_{0}\right\}, k\right) \rightarrow\left(X, k_{0}\right)$ is a $\left(k, k_{0}\right)$-isomorphism, and

(2) $\left.W_{Y}\right|_{\left\{x_{0}\right\} \times Y}:\left(\left\{x_{0}\right\} \times Y, k\right) \rightarrow\left(Y, k_{1}\right)$ is a $\left(k, k_{1}\right)$ isomorphism.

(W2) Any two distinct elements $x\left(\neq x_{0}\right) \in X \subset X \vee Y$ and $y\left(\neq y_{0}\right) \in Y \subset X \vee Y$ are not $k(m, n)$ (or $k$ )adjacent to each other.

Example 1. For several types of simple closed $k$-curves in $\mathbb{Z}^{n}, n \in\{2,3\}$, (see Fig. 1), we can observe the following compatible $k$-adjacencies of digital wedges: 
(1) $\left(M S C_{18} \vee S C_{8}^{2,4}, 18\right)$.

(2) $\left(S C_{8}^{2,6} \vee S C_{26}^{2,4}, 26\right)$.

(3) There is no compatible $k$-adjacency of $\left(M S C_{18} \mathrm{~V}\right.$ $\left.S C_{26}^{2,4}, k\right)$.

(4) There is no compatible $k$-adjacency of $\left(S C_{4}^{2,8} \mathrm{~V}\right.$ $\left.S C_{8}^{2,6}, k\right)$.

Proof. (1) and (2) are clearly proved.

(3) In terms of (W1) of Definition 10, $M S C_{18} \vee S C_{26}^{2,4}$ should be considered in $\mathbb{Z}^{3}$. Now we only examine if the digital wedge $M S C_{18} \vee S C_{26}^{2,4}$ has a compatible 26adjacency because 18 - and 6 -adjacencies cannot be valid contrary to (W1) of Definition 10. Suppose that it has a compatible 26 -adjacency. Then it clearly violates the property (W1-1) of Definition 10.

(4) With a method similar to the proof of (3), we can prove that $S C_{4}^{2,8} \vee S C_{8}^{2,6} \subset \mathbf{Z}^{2}$ cannot have any compatible $k$ adjacency, $k \in\{4,8\}$. Precisely, owing to the properties (W1) (resp. (W1-1)), $S C_{4}^{2,8} \vee S C_{8}^{2,6}$ cannot have the compatible 4-(resp., 8-)adjacency.

Remark 3. The notion of compatible adjacency of a digital wedge can be considered to be the most reasonable one of a digital wedge. In addition, there is no need for a uniqueness of a compatible adjacency of a digital wedge.

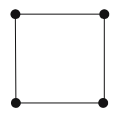

$\mathrm{SC}_{4}^{2,4}$

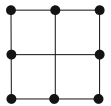

$\mathrm{SC}_{4}^{2,8}$

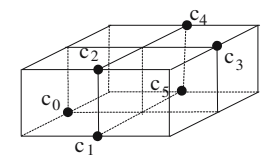

$\mathrm{MSC}_{18}$

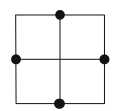

$\mathrm{SC}_{8}^{2,4}$

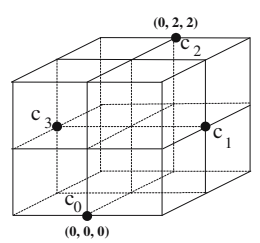

$\mathrm{SC}_{26}^{3,4}:=\mathrm{MSC}_{26}$
Fig. 1. Several kinds of simple closed $k$-curves (Han, 2005b; 2006d; 2010b).

In view of Example 1 and Remark 3, we obtain the following:

Remark 4. A compatible $k$-adjacency of a digital wedge has the following properties:

(1) By comparing with the $k$-adjacency of a wedge product Han $(2009 \mathrm{c})$, we can observe that the current compatible adjacency requires further (W1-1).

(2) Consider $S C_{k_{i}}^{n_{i}, l_{i}}$ with $k_{i}=3^{n_{i}}-1, i \in\{0,1\}$. Assume $n_{0} \leq n_{1}$. Then we always have a compatible $k_{1}$ adjacency of the digital wedge $S C_{k_{0}}^{n_{0}, l_{0}} \vee S C_{k_{1}}^{n_{1}, l_{1}} \subset \mathbb{Z}^{n_{1}}$.

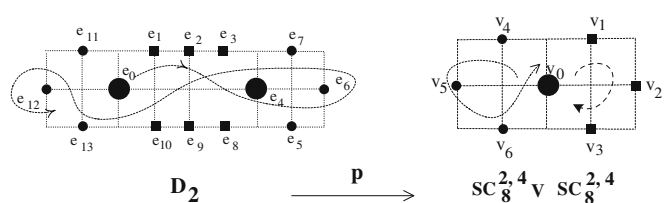

Fig. 2. (8, 8)-covering $\left(D_{2}, p, S C_{8}^{2,4} \vee S C_{8}^{2,4}\right)$, which does not satisfy a radius 2 local isomorphism (Han, 2009b).

(3) Consider $S C_{k_{i}}^{n_{i}, l_{i}}$ with $k_{i}\left(m_{i}, n_{i}\right)$-adjacency, $i \in$ $\{0,1\}$. Assume $n_{0} \leq n_{1}$. If $m_{0}=m_{1}$, then we always have a compatible $k\left(m_{1}, n_{1}\right)$-adjacency of the digital wedge $S C_{k_{0}}^{n_{0}, l_{0}} \vee S C_{k_{1}}^{n_{1}, l_{1}} \subset \mathbb{Z}^{n_{1}}$ (Han, 2009c).

\subsection{Limitation of a regular covering space in digital} covering theory. In spite of the study of various properties of a regular covering space in Section 3, in relation to the study of an automorphism group of a digital covering space, a digital regular covering space has a limitation. More precisely, as discussed in (1), (2), Theorems 1 and 2, the study of an automorphism group of a digital covering requires to satisfy a radius 2 local isomorphism of a given digital covering. Thus, if a $\left(k_{0}, k_{1}\right)$ covering does not satisfy a radius 2 local isomorphism, then we have an obstacle to the study of digital homotopic properties of a digital covering as well as its automorphism group (see Boxer, 2006; Boxer and Karaca, 2008; Han, 2005b; 2006b). To be specific, let us now consider the two $(8,8)$-coverings $\left(S C_{8}^{2,12}, p, S C_{8}^{2,4}\right)$ and $\left(D_{2}, p, S C_{8}^{2,4} \vee S C_{8}^{2,4}\right)$ in Fig. 2, in which none of them can satisfy a radius 2 local isomorphism. In other words, the digital covering $\left(S C_{8}^{2,12}, p, S C_{8}^{2,4}\right)$ cannot be a radius 2-(8,8)-covering. Namely, in Fig. 2, assume that $p: D_{2} \rightarrow S C_{8}^{2,4} \vee S C_{8}^{2,4}$ maps each of solid squares, small solid circles and big solid circles of $D_{2}$ into the corresponding ones in $S C_{8}^{2,4} \vee S C_{8}^{2,4}$. Then we can observe that $\left(D_{2}, p, S C_{8}^{2,4} \vee S C_{8}^{2,4}\right)$ cannot satisfy a radius 2 local isomorphism, either. Consequently, we have to establish another notion instead of a regular $\left(k_{0}, k_{1}\right)$-covering (see Section 5).

\section{Ultra regular $\left(k_{0}, k_{1}\right)$-covering space and its automorphism group}

In this section we develop the notion of an ultra regular covering space and investigate its properties related to the study of its automorphism group. As discussed in Section 3 , for a radius 2 covering $(E, p, B)$, its automorphism group was studied by Han (2008a) (see also Boxer and Karaca, 2008; Han, 2009b). Meanwhile, if a digital covering $(E, p, B)$ does not satisfy a radius 2 local isomorphism (see Fig. 2), then its automorphism group has intrinsic features, which remains to be studied. In addition, for a digital covering space which satisfies a radius 2 local 
isomorphism, its automorphism group can also be studied (see Example 2(2)). Thus, in order to study this problem, this section establishes the notion of an ultra regular digital covering space and studies its automorphism group, which can play an important role in classifying digital covering spaces. Motivated by the transitivity of an automorphism of a covering space found work of in Massey (1977), we can define the following.

Definition 11.

For a $\left(k_{0}, k_{1}\right)$-covering $\left(\left(E, e_{0}\right), p,\left(B, b_{0}\right)\right)$, we say that $\operatorname{Aut}(E \mid B)$ acts transitively on $p^{-1}\left(b_{0}\right)$ if for any two distinct points $e_{0}$ and $e_{1}$ in $p^{-1}\left(b_{0}\right)$ there is $\phi \in A u t(E \mid B)$ such that $\phi\left(e_{0}\right)=e_{1}$.

In general, for a $\left(k_{0}, k_{1}\right)$-covering $\left(\left(E, e_{0}\right), p\right.$, $\left.\left(B, b_{0}\right)\right) \operatorname{Aut}(E \mid B)$ need not act transitively on $p^{-1}\left(b_{0}\right)$ (see Example 2(1)).

\section{Example 2.}

(1) Consider the map $p_{1}: E_{1} \rightarrow S C_{8}^{2,6} \vee S C_{8}^{2,6}$ given by Fig. 3(a). To be specific, assume that $p_{1}$ maps each of solid squares, small solid circles, and big solid circles of $E_{1}$ into the corresponding ones in $S C_{8}^{2,6} \vee S C_{8}^{2,6}$ along with the arrows in $E_{1}$. Then, for the $(8,8)$-covering $\left(E_{1}, p_{1}, S C_{8}^{2,6} \vee S C_{8}^{2,6}\right)$, we can observe that $\operatorname{Aut}\left(E_{1} \mid S C_{8}^{2,6} \vee S C_{8}^{2,6}\right)$ cannot act transitively on $p^{-1}\left(v_{0}\right)$ for the point $v_{0} \in S C_{8}^{2,6} \vee S C_{8}^{2,6}$ (see Fig. 3(a)). More precisely, for two distinct points $e_{i}$ and $e_{j}$ in $p^{-1}\left(v_{0}\right)$ (see the points $(0,0),(6,1)$ in $p^{-1}\left(v_{0}\right)$ ), there is no $\phi \in \operatorname{Aut}\left(E_{1} \mid S C_{8}^{2,6} \vee S C_{8}^{2,6}\right)$ such that $\phi\left(e_{i}\right)=e_{j}$.

(2) Consider the map $p_{2}: E_{2} \rightarrow S C_{8}^{2,6} \vee S C_{8}^{2,6}$ given by Fig. 3(b). Then the $(8,8)$-covering $\left(E_{2}, p_{2}, S C_{8}^{2,6} \mathrm{~V}\right.$ $\left.S C_{8}^{2,6}\right)$ is a radius $2-(8,8)$-covering. For any two distinct points $e_{i}$ and $e_{j}$ (e.g., the points $e_{0}, e_{6} \in p^{-1}\left(v_{0}\right) \subset E_{2}$ ), there is always $\phi \in \operatorname{Aut}\left(E_{2} \mid S C_{8}^{2,6} \vee S C_{8}^{2,6}\right)$ such that $\phi\left(e_{i}\right)=e_{j}$.

In view of Example 2, we can clearly observe that, for a $\left(k_{0}, k_{1}\right)$-covering map $p:\left(E, e_{0}\right) \rightarrow\left(B, b_{0}\right)$ and two distinct points $e_{0}$ and $e_{1}$ in $p^{-1}\left(b_{0}\right)$, there may not be an element $\phi \in \operatorname{Aut}(E \mid B)$ such that $\phi\left(e_{0}\right)=e_{1}$. Unlike Example 2, motivated by Lemma 8.1 of Massey (1977), by Theorem 2 , we clearly obtain the following.

Lemma 4. If a radius 2 - $\left(k_{0}, k_{1}\right)$-covering map $p$ : $\left(E, e_{0}\right) \rightarrow\left(B, b_{0}\right)$ is regular, then $\operatorname{Aut}(E \mid B)$ acts transitively on $p^{-1}\left(b_{0}\right)$.

For a $\left(k_{0}, k_{1}\right)$-covering map $p:\left(E, e_{0}\right) \rightarrow\left(B, b_{0}\right)$ which does not satisfy a radius 2 local isomorphism, the study of $\operatorname{Aut}(E \mid B)$ remains to be approached. In order to deal with this problem, we need to make the version of Han (2006b) advanced into the following notion, which is different from a regular $\left(k_{0}, k_{1}\right)$-covering of Han (2006b).

Definition 12. A $\left(k_{0}, k_{1}\right)$-covering $\left(\left(E, e_{0}\right), p,\left(B, b_{0}\right)\right)$ is called an ultra regular (briefly, UR-) $\left(k_{0}, k_{1}\right)$-covering if $\operatorname{Aut}(E \mid B)$ acts transitively on $p^{-1}\left(b_{0}\right)$.
Let us now study an important property of a UR$\left(k_{0}, k_{1}\right)$-covering which proposes a method of determining if a $\left(k_{0}, k_{1}\right)$-covering is a UR- $\left(k_{0}, k_{1}\right)$-one. The following theorem can characterize a UR- $\left(k_{0}, k_{1}\right)$-covering.

Theorem 3. The following are equivalent:

(1) $A\left(k^{\prime}, k\right)$-covering $\left.\left(\left(E, e_{0}\right)\right), p,\left(B, b_{0}\right)\right)$ is ultra regular.

(2) For a $\left(k^{\prime}, k\right)$-covering $\left.\left(\left(E, e_{0}\right)\right), p,\left(B, b_{0}\right)\right)$ assume a closed $k$-curve $\alpha:[0, m]_{\mathbb{Z}} \rightarrow(B, k)$ with $\alpha(0)=b_{0} \in$ $B$. Either each of all liftings of $\alpha$ on $\left(E, k^{\prime}\right)$ is a $k^{\prime}$-closed curve or none of them is a $k^{\prime}$-closed one.

Proof.

Case 1. Let us assume that a given covering $\left.\left(\left(E, e_{0}\right)\right), p,\left(B, b_{0}\right)\right)$ is a radius $2-\left(k^{\prime}, k\right)$-covering. Then the assertion is clear. Precisely, in this case, since the property (2) of the current theorem implies that for any points $e_{0} \in p^{-1}\left(b_{0}\right)$ the subgroups $p_{*} \pi^{k^{\prime}}\left(E, e_{0}\right) \subset$ $\pi^{k}\left(B, b_{0}\right)$ are normal, they are the same. Finally, by Theorem 1(2), we can observe that the given $\left(k^{\prime}, k\right)$-covering $(E, p, B)$ is regular. Consequently, by Lemma 4, the proof is completed.

Case 2. Let us now prove the case that a given covering $\left.\left(\left(E, e_{0}\right)\right), p,\left(B, b_{0}\right)\right)$ is not a radius $2-\left(k^{\prime}, k\right)$-covering. $(1) \Rightarrow(2)$ : For a closed $k$-curve $\alpha:[0, m]_{\mathbb{Z}} \rightarrow(B, k)$ with $\alpha(0)=b_{0} \in B$, assume that there are digital liftings of $\alpha$ on $\left(E, k^{\prime}\right)$ of which one of them is a $k^{\prime}$-closed curve and another of them is a $k^{\prime}$-path that is not a $k^{\prime}$-closed curve. Then the given $\left(k^{\prime}, k\right)$-covering $(E, p, B)$ cannot be a UR- $\left(k^{\prime}, k\right)$-covering because $A u t(E \mid B)$ cannot act transitively on $p^{-1}\left(b_{0}\right)$. For instance, owing to the point $(0,0) \in p^{-1}\left(v_{0}\right)$, the digital covering $\left(E_{1}, p_{1}, S C_{8}^{2,6} \vee\right.$ $\left.S C_{8}^{2,6}\right)$ in Fig. 3(a) cannot be a UR- $(8,8)$-covering.

$(2) \Rightarrow(1)$ : For a $\left(k^{\prime}, k\right)$-covering $(E, p, B)$ satisfying the property (2) we can clearly observe that $A u t(E \mid B)$ acts transitively on $p^{-1}\left(b_{0}\right)$, which completes the proof. For instance, see the $(8,8)$-covering $\left(D_{2}, p, S C_{8}^{2,4} \vee S C_{8}^{2,4}\right)$ in Fig. 2.

Due to Theorem 3, hereafter, regardless of the requirement of a radius 2 local isomorphism of a $\left(k^{\prime}, k\right)$ covering, we can have a very convenient method of determining if a given digital covering is UR- $\left(k^{\prime}, k\right)$ regular. Thus, hereafter, by using Theorem 3 , for a $\left(k^{\prime}, k\right)$ covering $(E, p, B)$ we can study $A u t(E \mid B)$ without using digital homotopic tools.

By Theorem 1, we obtain the following.

\section{Corollary 1.}

(1) For any $S C_{k}^{n, l}$ the $(2, k)$-covering map $p: \mathbb{Z} \rightarrow$ $S C_{k}^{n, l}:=\left(c_{t}\right)_{t \in[0, l-1]_{\mathbf{z}}}$ given by $p(t)=c_{t(\bmod l)}, t \in \mathbb{Z}$ is ultra regular.

(2) The map $p: S C_{k}^{n, m l}:=\left(a_{t}\right)_{t \in[0, m l-1]_{\mathbf{z}}} \rightarrow S C_{k}^{n, l}:=$ $\left(c_{t}\right)_{t \in[0, l-1]_{\mathbb{Z}}}$ given by given by $p\left(a_{i}\right)=c_{i(\bmod l)}, m \in \mathbb{N}$ is ultra regular, where $m \in \mathbb{N}$ and $S C_{k}^{n, l}$ need not be $k$ contractible. 

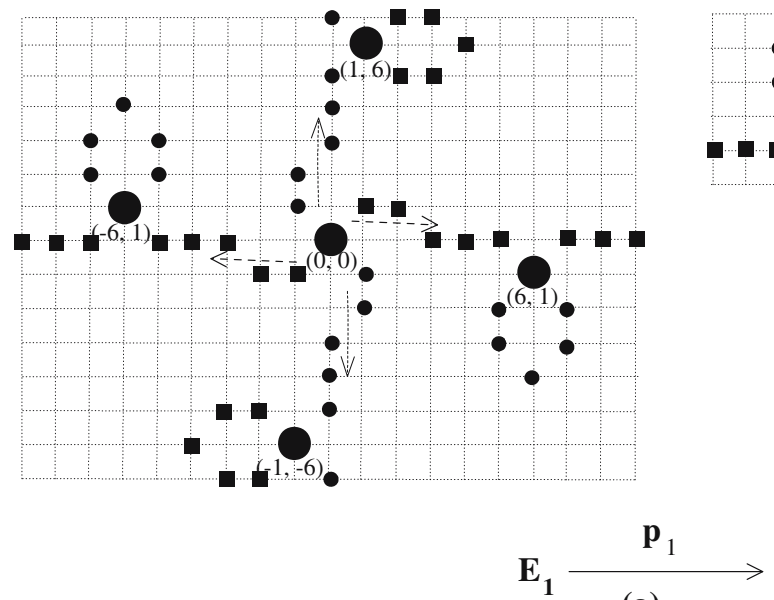

(a)

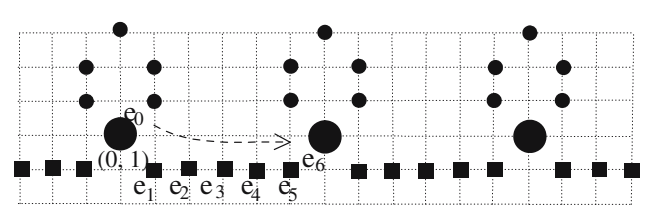

$\mathbf{p}_{2} \mathbf{E}_{2}$

(b)

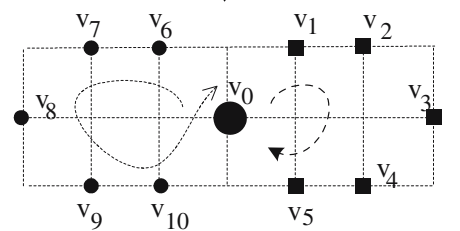

$\operatorname{SC}_{8}^{2,6} \mathrm{~V} \operatorname{SC}_{8}^{2,6}$

Fig. 3. Infinite fold $(8,8)$-covering $\left(E_{1}, p_{1}, S C_{8}^{2,6} \vee S C_{8}^{2,6}\right)$ (Han, 2005c), which does not allow an automorphism with transitive action (a); infinite fold $(8,8)$-covering $\left(E_{2}, p_{2}, S C_{8}^{2,6} \vee S C_{8}^{2,6}\right)$ from the work of Boxer (2006), which is both ultra regular and regular (b).

(3) The map $p: D_{2} \rightarrow S C_{8}^{2,4} \vee S C_{8}^{2,4}$ in Fig. 2 is a UR- $(8,8)$-covering.

\section{Proof.}

(1) Take a base point $c_{0} \in S C_{k}^{n, l}$. Then we can obtain $p^{-1}\left(c_{0}\right):=l \mathbb{Z} \subset \mathbb{Z}$. By Theorem 3 , we can clearly observe that $\operatorname{Aut}(E \mid B):=l \mathbb{Z}$ acts transitively on $p^{-1}\left(c_{0}\right)$.

(2) By a similar method as the proof of (1), the proof can be clearly completed by using the circulation on $S C_{k}^{n, m l}$ depending on the points $p^{-1}\left(c_{0}\right)$.

(3) The assertion follows from Theorem 3.

\section{Merits of an ultra regular covering space}

For a digital space $\left(X, k_{1}\right)$, how can we describe a difference between a UR- $\left(k_{0}, k_{1}\right)$-covering and a regular $\left(k_{0}, k_{1}\right)$-covering over $\left(X, k_{1}\right)$ ? In the light of Theorem 3 , this section discusses some merits of a UR- $\left(k_{0}, k_{1}\right)$ covering. In Section 3 , for a radius $2-\left(k_{0}, k_{1}\right)$-covering, its regularity has been studied. But a UR- $\left(k_{0}, k_{1}\right)$-covering need not require a radius 2 local isomorphism. In view of this difference, we can observe that a UR- $\left(k_{0}, k_{1}\right)$ covering has strong merits of classifying digital covering space. By comparing an ultra regular covering space with a regular covering space, we obtain the following.

\section{Theorem 4.}

(1) A regular $\left(k_{0}, k_{1}\right)$-covering space does not imply a $U R$ - $\left(k_{0}, k_{1}\right)$-covering space.

(2) For a digital space $\left(X, k_{1}\right)$, let $R_{2}(X)$ denote the set of all radius $2-\left(k_{0}, k_{1}\right)$-coverings over $\left(X, k_{1}\right)$. Then we obtain the following: In $R_{2}(X)$ a UR- $\left(k_{0}, k_{1}\right)$-covering is equivalent to a regular $\left(k_{0}, k_{1}\right)$-covering.

\section{Proof.}

(1) As an example, consider the infinite fold $(8,8)$ - covering map $p: E_{5} \rightarrow S C_{8}^{2,4} \vee S C_{8}^{2,4}$ in Fig. 4(c). Precisely, assume that $p_{5}$ maps each of solid squares, small solid circles, and big solid circles of $E_{5}$ into the corresponding ones in $S C_{8}^{2,4} \vee S C_{8}^{2,4}$. Since both $\pi^{8}\left(E_{5}\right)$ and $\pi^{8}\left(S C_{8}^{2,4} \vee S C_{8}^{2,4}\right)$ are trivial, $\left(E_{5}, p_{5}, S C_{8}^{2,4} \vee S C_{8}^{2,4}\right)$ is a regular $(8,8)$-covering. But it cannot be a UR- $(8,8)$ covering because $\operatorname{Aut}\left(E_{5}, S C_{8}^{2,4} \vee S C_{8}^{2,4}\right)$ is trivial. Precisely, the $(8,8)$-covering $\left(E_{5}, p, S C_{8}^{2,4} \vee S C_{8}^{2,4}\right)$ has the only digital isomorphism such as the identity. To be specific, if there is a digital automorphism $h: E_{5} \rightarrow E_{5}$, then any 8-loop in $S C_{8}^{2,4} \vee S C_{8}^{2,4}$ that lifts to an 8-loop in $E_{5}$ at $(0,0)$ also lifts to an 8-loop when the lift begins at $h(0,0)$. In this case we clearly observe that $h$ is exactly the identity.

(2) In $R_{2}(X)$, by Theorem 2 and Lemma 4, it is clear that a $\mathrm{UR}-\left(k_{0}, k_{1}\right)$-covering $(E, p, X)$ is equivalent to a regular $\left(k_{0}, k_{1}\right)$-covering.

In the light of Theorem 4 , if a $\left(k_{0}, k_{1}\right)$-covering is not a radius $2-\left(k_{0}, k_{1}\right)$-covering, then a comparison between an ultra regular covering space and a regular covering space depends on the situation. To study a UR$\left(k_{0}, k\right)$-covering $\left(\left(E, k_{0}\right), p, S C_{k_{1}}^{n_{1}, l_{1}} \vee S C_{k_{2}}^{n_{2}, l_{2}}\right)$ up to a digital covering isomorphism, let us recall that the $k$ fundamental group of $\left(S C_{k}^{n, l_{0}} \vee S C_{k}^{n, l_{1}}, k\right)$ is a free group with two generators (Han, 2005c) (see also Han, 2007a), where $S C_{k}^{n, l_{i}}$ is not $k$-contractible, $i \in\{0,1\}$ and the $k$-adjacency of $\left.S C_{k_{1}}^{n_{1}, l_{1}} \vee S C_{k_{2}}^{n_{2}, l_{2}}\right)$ is assumed to be compatible according to Definition 10. By using this property and Theorem 3, let us demonstrate Theorem 4 with the following examples.

Example 3. For illustration, consider the $(8,8)$-covering $\left(E_{3}, p_{3}, S C_{8}^{2,4} \vee S C_{8}^{2,4}\right)$ in Fig. 4(a), which is not a radius $2-(8,8)$-covering. Then we can observe that it is not a 

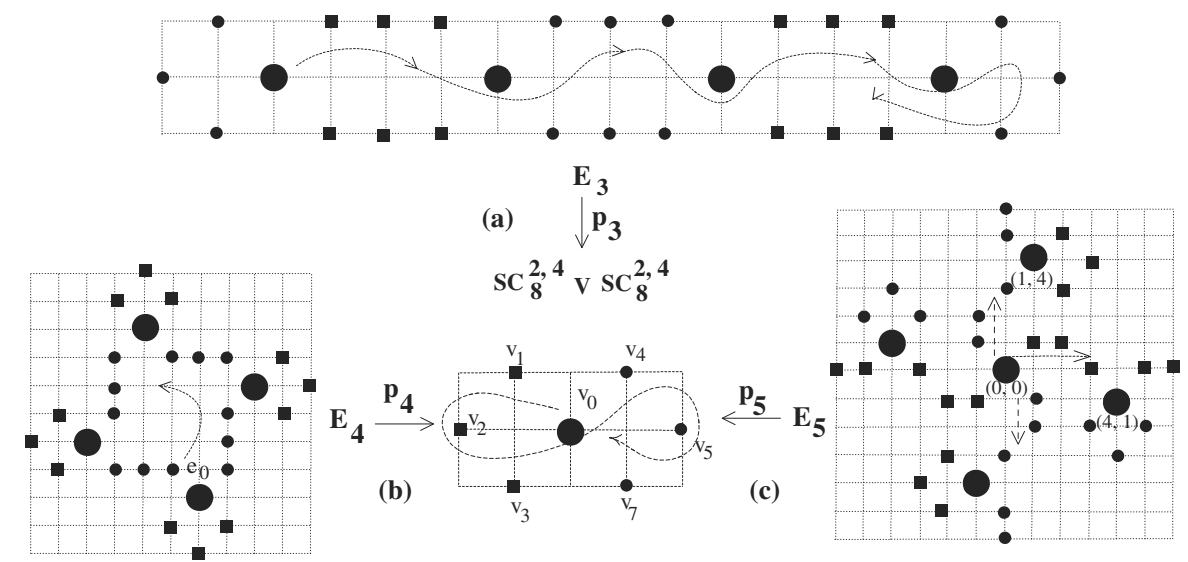

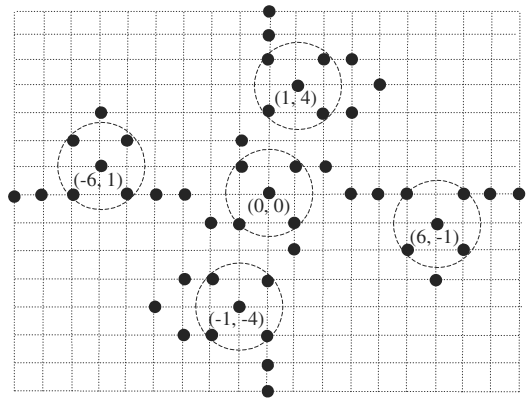

$\mathrm{U}_{1}$ of Fig. 6 by Han (2010a)

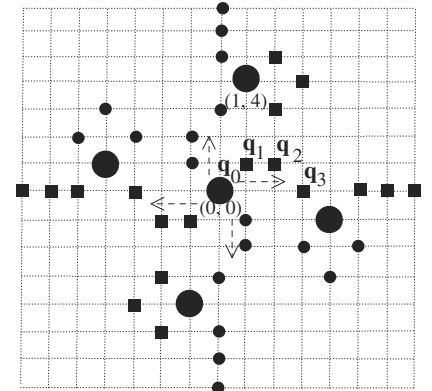

$E_{1}$ of Fig. 1 by Han (2009c)

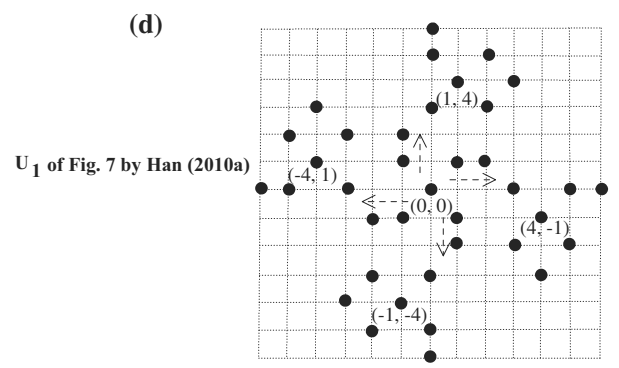

Fig. 4. 4-fold (8,8)-covering $\left(E_{3}, p_{3}, S C_{8}^{2,4} \vee S C_{8}^{2,4}\right)$, which is not UR-regular (a); 4-fold (8, 8)-covering $\left(E_{4}, p_{4}, S C_{8}^{2,4} \vee S C_{8}^{2,4}\right)$ found in the work of Han (2009c), which is UR-regular (b); infinite fold $(8,8)$-covering $\left(E_{5}, p_{5}, S C_{8}^{2,4} \vee S C_{8}^{2,4}\right)$, which is not UR-regular but regular (c); correction of Fig. $E_{1}$ and $U_{1}$ presented by Han (2009c; 2010a) (d).

UR-(8, 8)-covering.

By Theorem 4(2), we can observe the following.

\section{Example 4.}

(1) Consider the $(8,8)$-covering $\left(E_{4}, p_{4}, S C_{8}^{2,4} \vee S C_{8}^{2,4}\right)$ in Fig. 4(b), which is not a radius $2-(8,8)$-covering. Then we can observe that it is a UR- $(8,8)$-covering.

(2) Consider the $(8,8)$-covering $\left(E_{5}, p_{5}, S C_{8}^{2,4} \vee S C_{8}^{2,4}\right)$ in Fig. $4(\mathrm{c})$, which is not a radius $2-(8,8)$-covering. Then we can observe that it is not a UR- $(8,8)$-covering but a regular $(8,8)$-covering.

Remark 5. In relation to the study of an automorphism group of a $\left(k_{0}, k_{1}\right)$-covering, since Theorem 3 does not require a radius 2 local $\left(k_{0}, k_{1}\right)$-isomorphism of the given digital covering as well as digital homotopic properties such as Theorem 2, the notion of a UR- $\left(k_{0}, k_{1}\right)$-covering is so useful (see Theorem 3).

Remark 6. (Correcting) Since the two objects $U_{1}$ of Fig. 6 and $U_{1}$ of Fig. 7 found in the work of Han (2010a) are misprinted at the point $(0,0) \in \mathbb{Z}^{2}$, we can now correct them (see Fig. 4(d)). With the same criterion, the objects $E_{1}$ of Fig. 1 found in the paper by Han (2009c) should be corrected at the point $(0,0) \in \mathbb{Z}^{2}$ (motivated by Fig. 4 of Han (2005c)).

\section{Concluding remarks and further work}

In relation to the study of an automorphism group of a digital covering and the classification of digital spaces, the 
paper has studied the following. First, we have established the notion of compatible adjacency of a digital wedge which can be used for studying an automorphism group of a digital covering. Second, we have established the notion of a UR- $\left(k^{\prime}, k\right)$-covering space and investigated some properties of an ultra regular covering space over $S C_{k}^{n, l}$ or $S C_{k_{1}}^{n_{1}, l_{1}} \vee S C_{k_{2}}^{n_{2}, l_{2}}$. Then, by comparing an ultra regular covering with a regular covering, we have proposed some merits of an ultra regular covering. Also, by using the GU- $\left(k^{\prime}, k\right)$-covering property of $\left(E, p, S C_{k}^{n, l}\right)$, where $\left(E, k^{\prime}\right)$ is $\left(k^{\prime}, 2\right)$-isomorphic to $(\mathbb{Z}, 2)$, we have demonstrated that an automorphism group of a $\left(k^{\prime}, k\right)$-covering map can be strongly used in the classification of digital covering spaces over $S C_{k}^{n, l}$ or a digital wedge without any limitation of a radius 2 local isomorphism of a digital covering.

\section{Acknowledgment}

This research was supported by the Basic Science Research Program through the National Research Foundation of Korea (NRF) funded by the Ministry of Education, Science and Technology (2008C00119). This paper was supported by the selection of a research-oriented professor of Chonbuk National University in 2010.

The author wishes to thank the anonymous referees who made careful corrections and suggested many meaningful ideas.

\section{References}

Boxer, L. (1999). A classical construction for the digital fundamental group, Journal of Mathematical Imaging and Vision 10(1): 51-62.

Boxer, L. (2006). Digital products, wedge, and covering spaces, Journal of Mathematical Imaging and Vision 25(2): 159_ 171.

Boxer, L. and Karaca, I. (2008). The classification of digital covering spaces, Journal of Mathematical Imaging and Vision 32(1): 23-29.

Han, S.E. (2003). Computer topology and its applications, Honam Mathematical Journal 25(1): 153-162.

Han, S.E. (2005a). Algorithm for discriminating digital images w.r.t. a digital $\left(k_{0}, k_{1}\right)$-homeomorphism, Journal of Applied Mathematics and Computing 18(1-2): 505-512.

Han, S.E. (2005b). Digital coverings and their applications, Journal of Applied Mathematics and Computing 18(1-2): 487-495.

Han, S.E. (2005c). Non-product property of the digital fundamental group, Information Sciences 171 (1-3): 73-91.

Han, S.E. (2005d). On the simplicial complex stemmed from a digital graph, Honam Mathematical Journal 27(1): 115129.

Han, S.E. (2006a). Connected sum of digital closed surfaces, Information Sciences 176(3): 332-348.
Han, S.E. (2006b). Discrete Homotopy of a Closed $k$-Surface, Lecture Notes in Computer Science, Vol. 4040, SpringerVerlag, Berlin, pp. 214-225.

Han, S.E. (2006c). Erratum to "Non-product property of the digital fundamental group", Information Sciences 176(1): 215-216.

Han, S.E. (2006d). Minimal simple closed 18-surfaces and a topological preservation of 3D surfaces, Information Sciences 176(2): 120-134.

Han, S.E. (2007a). Strong $k$-deformation retract and its applications, Journal of the Korean Mathematical Society 44(6): 1479-1503.

Han, S.E. (2007b). The $k$-fundamental group of a closed $k$ surface, Information Sciences 177(18): 3731-3748.

Han, S.E. (2008a). Comparison among digital fundamental groups and its applications, Information Sciences 178(8): 2091-2104.

Han, S.E. (2008b). Equivalent $\left(k_{0}, k_{1}\right)$-covering and generalized digital lifting, Information Sciences 178(2): 550-561.

Han, S.E. (2008c). Map preserving local properties of a digital image, Acta Applicandae Mathematicae 104(2): 177-190.

Han, S.E. (2008d). The $k$-homotopic thinning and a torus-like digital image in $\mathbf{Z}^{n}$, Journal of Mathematical Imaging and Vision 31(1): 1-16.

Han, S.E. (2009a). Cartesian product of the universal covering property, Acta Applicandae Mathematicae 108(2): 363383.

Han, S.E. (2009b). Regural covering space in digital covering theory and its applications, Honam Mathematical Journal 31(3): 279-292.

Han, S.E. (2009c). Remark on a generalized universal covering space, Honam Mathematical Journal 31(3): 267-278.

Han, S.E. (2010a). Existence problem of a generalized universal covering space, Acta Applicandae Mathematicae 109(3): 805-827.

Han, S.E. (2010b). Multiplicative property of the digital fundamental group, Acta Applicandae Mathematicae 110(2): 921-944.

Han, S.E. (2010c). KD-( $\left.k_{0}, k_{1}\right)$-homotopy equivalence and its applications, Journal of the Korean Mathematical Society 47(5): 1031-1054.

Han, S.E. (2010d). Properties of a digital covering space and discrete Deck's transformation group, The IMA Journal of Applied Mathematics, (submitted).

Khalimsky, E. (1987). Motion, deformation, and homotopy in finite spaces, Proceedings of IEEE International Conferences on Systems, Man, and Cybernetics, pp. 227-234.

Kim I.-S., and Han, S.E. (2008). Digital covering theory and its applications, Honam Mathematical Journal 30(4): 589602.

Kong, T.Y. and Rosenfeld, A. (1996). Topological Algorithms for the Digital Image Processing, Elsevier Science, Amsterdam. 
Malgouyres, R. and Lenoir, A. (2000). Topology preservation within digital surfaces, Graphical Models 62(2): 71-84.

Massey, W.S. (1977). Algebraic Topology, Springer-Verlag, New York, NY.

Rosenfeld, A. (1979). Digital topology, American Mathematical Monthly 86: 76-87.

Rosenfeld, A. and Klette, R. (2003). Digital geometry, Information Sciences 148: 123-127.

Spanier, E.H. (1966). Algebraic Topology, McGraw-Hill Inc., New York, NY.

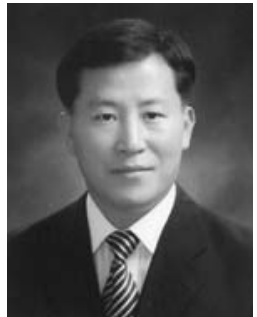

Sang-Eon Han is a professor (Ph.D.) working at Chonbuk National University. His research is on digital topology, digital geometry and pure topology. He holds a Bachelor's and a Master's degree in mathematics from Chonbuk National University and $\mathrm{Ph} . \mathrm{D}$. in mathematics from Chonnam National University. He has been a visiting scholar at the Department of Mathematics, Stanford University. He has developed digital covering theory. Indeed, the theory can be substantially used in the fields of both digital topology and digital geometry. $\mathrm{He}$ has received an academic award from the Honam Mathematical Society and his academic activities were introduced in the 2008 edition of Who's Who in the World.
Received: 10 January 2010

Revised: 10 May 2010 